\title{
A self-closed system of equations of damage evolution*
}

\author{
Y.L. BAI, M.F. XIA ${ }^{1}$, F.J. KE ${ }^{2}$, W.S. HAN and B. FANG \\ LNM, Institute of Mechanics, Chinese Academy of Sciences, Beijing 100080, People's Republic of China \\ 'Department of Physics, Peking University, Beijing 100871, People's Republic of China \\ ${ }^{2}$ Department of Applied Mathematics and Physics, Beijing University of Aeronautics and Astronautics, \\ Beijing 100083, People's Republic of China
}

Received 5 December 1994; accepted in revised form 10 November 1995

Abstract. This paper introduces a statistical mesomechanical approach to the evolution of damage. A self-closed formulation of the damage evolution is derived.

\section{Introduction}

The concept of damage was introduced by Kachanov [1]. According to his approach, a damage variable $0<D \leqslant 1$ was defined and an a priori evolution law was assumed as

$$
\dot{D} \sim \frac{1}{(1-D)^{\gamma}}
$$

A plain interpretation of damage is a certain properly averaged amount of broken microscopic elements. So, a simple and widely applied correlation of actual stress $\sigma$ on the ligaments still supporting load with damage $D$ is presumed as

$$
\sigma=\frac{\sigma_{0}}{(1-D)}
$$

where $\sigma_{0}$ is the nominal stress. In this way, the damage variable was included in constitutive formulation as an internal variable describing a kinetic process of microscopic transformations in damaged materials. Obviously, this approach to damage and failure of materials is decisively based upon the presumptive model (1), which needs a sound physical basis.

On the other hand, Cocks and Ashby [2] assumed a mesomechanical approach to the evolution of damage in creep. The essential idea is to isolate a cylindrical element of material (about the void spacing) centred on a void.

Actually, macroscopic damage should be a collective response of microdamage. Therefore, one should understand how microdamage evolves collectively, i.e. in which situation an average approach works, what is the physical basis for a widely assumed semi-empirical equation of damage, how and when disorder in microscopic structure and damage must be taken into account, and so on. In the light of a statistical mesomechanical study of damage we intend to provide some of our results concerning some of these questions. The effect of mesoscopic disorder and fluctuation on damage and failure of materials can be referred in our other papers $[3,4]$.

\footnotetext{
* Presented at the Far East Fracture Group (FEFG) International Symposium on Fracture and Strength of Solids, 4-7 July 1994 in Xi'an, China.
} 


\section{Statistical mesomechanical equations of damage evolution}

It has been found that evolution of microdamage can be depicted by the following conservative equation in two-dimensional phase space $\left(c, c_{0}\right)[5-7]$

$$
\frac{\partial n_{0}}{\partial t}+\frac{\partial\left(n_{0} V\right)}{\partial c}=n_{0 N}
$$

where $n_{0}=n_{0}\left(t, c, c_{0}, \sigma\right)$ is the number density of microdamage with current and initial size $c$ and $c_{0}$ respectively, and $t$ is the generalized time.

$$
n_{0 N}=n_{N}\left(c_{0}, \sigma\right) \delta\left(c-c_{0}\right),
$$

is the nucleation rate of microdamage and

$$
V=V\left(c, c_{0}, \sigma\right)
$$

is the law of growth rate of microdamage. In this formulation, the concept of ideal microdamage is assumed, the coalescence of microdamage is ignored but the interaction of microdamage can be considered by the variation of the stress field [6], like expression (2). Equations (3)-(5) constitute the statistical description of evolution of microdamage.

The solution to (3)-(5) was obtained under constant stress $\sigma$ [7]

$$
n_{0}\left(t, c, c_{0}, \sigma\right)=\frac{n_{N}\left(c_{0}, \sigma\right)}{V\left(c, c_{0}, \sigma\right)},
$$

because the initial size $c_{0}$ of microdamage is an undetectable variable in experiments, the current number density $n$ can be defined and expressed as $[6,7]$

$$
n(t, c, \sigma)=\int_{\zeta}^{c} n_{0}\left(t, c, c_{0}, \sigma\right) \mathrm{d} c_{0}=\int_{\zeta}^{c} \frac{n_{N}\left(c_{0}, \sigma\right)}{V\left(c, c_{0}, \sigma\right)} \mathrm{d} c_{0},
$$

where $\zeta=\zeta(t, c)$ denotes the minimum initial size of microdamage of current size $c$ at time $t$ and is defined by the lower limit of an integral

$$
t=\int_{\zeta}^{c} \frac{\mathrm{d} c^{\prime}}{V\left(c^{\prime}, \zeta, \sigma\right)} \quad \text { or } \quad t=\int_{c_{0},}^{c_{f}} \frac{\mathrm{d} c^{\prime}}{V\left(c^{\prime}, c_{0}, \sigma\right)},
$$

where $c_{f}=c_{f}\left(t, c_{0}\right)$ is the front of the number density of microdamage in phase space.

\section{Evolution equation of continuum damage}

In accord with the physical interpretation of damage, the $i$ th order momentum of continuum damage can be expressed by number density of microdamage as

$$
D_{j}=\int_{0}^{\infty} c^{j} n(t, c, \sigma) \mathrm{d} c .
$$

Substitution of the solution (7) into expression (9) and exchanging the order of integrations leads to the continuum damage $D_{j}$ and its rate $\dot{D}_{j}$

$$
D_{j}(t, \sigma)=\int_{0}^{\infty} n_{N}\left(c_{0}, \sigma\right) \int_{c_{0}}^{c_{f}} \frac{c^{j} \mathrm{~d} c}{V\left(c, c_{0}, \sigma\right)} \mathrm{d} c_{0},
$$




$$
\dot{D}_{j}(t, \sigma)=\int_{0}^{\infty} n_{N}\left(c_{0}, \sigma\right) c_{f}^{j} \mathrm{~d} c_{0}=D_{N_{j}}+j \int_{0}^{\infty} \mathrm{d} c_{0} \int_{c_{0}}^{\infty} c^{j-1} V n_{0} \mathrm{~d} c
$$

where

$$
D_{N_{j}}(\sigma)=\int_{0}^{\infty} n_{N}\left(c_{0}, \sigma\right) c_{0}^{j} \mathrm{~d} c_{0}
$$

From expression (11), one can see that the rate of continuum damage $\dot{D}$ is dependent on the propogation of the front $c_{f}(t)$ of the number density of microdamage in phase-space. Generally speaking, there is not a simple relation between the continuum damage $D$ and its rate $\dot{D}$.

The application to practice of the evolution equation of damage (10), (11) and (9) needs details of meso-kinetical laws of microdamage, (4) and (5). Usually, it is desirable to find a self-closed equation of continuum damage, in which only a few macroscopically measurable parameters are included while detailed meso-kinetics are not needed.

One possibility can be deduced by adopting a viscous growth law of microdamage

$$
V\left(c, c_{0}, \sigma\right)=\beta c .
$$

After substituting (13) into (11), we obtain

$$
\dot{D}_{j}=\dot{D}_{j}\left(D_{j}, \sigma\right)=j \beta D_{j}+D_{N_{j}} \text {. }
$$

In expression (13), $\dot{D}_{j}$ is no longer dependent on time $t$ explicitly. So it is autonomous; in fact, (13) is exactly that which Davison and Stevens had suggested [8] by assuming a Taylor expansion.

A physical basis for growth rule (13) is viscous extension of microdamage [9]

$$
V\left(c, c_{0}, \sigma\right)=\frac{\sigma-\sigma_{*}}{\eta} c
$$

where $\eta$ is the viscous coefficient, and $\sigma_{*}$ is a threshold stress.

\section{Self-consistency of average stress}

As a matter of fact, stress must sustain simultaneous variation with continuum damage, like expression (2). Then, how to consider this effect?

When we re-examined the procedure, we developed a new point of view. If we intend to understand the mechanism of damage evolution, the solution of the number density of microdamage to (3)-(5) is a necessity. However, since engineers mainly have an eye to continuum damage, it might be possible for us to skip the derivation of the solution.

Noticing the definition (7) and integrating (3), we have

$$
\frac{\partial n}{\partial t}+\frac{\partial(n A)}{\partial c}=n_{N}(c, \sigma)
$$

where $A=\int_{0}^{\infty} n_{0} \cdot V \mathrm{~d} c_{0} / n$. Differentiation of (9) and substitution of (16) into it leads to

$$
\dot{D}_{j}=D_{N_{j}}+j \int_{0}^{\infty} A \cdot n(t, c, \sigma) \cdot c^{j-1} \mathrm{~d} c .
$$


Still, provided (13) or (15) works, (17) is reduced to (14). In this derivation, stress can be a variable parameter. So, substitution of (2) in (17) or (13) gives

$$
\dot{D}=D_{N}+J \cdot \beta D
$$

where $J$ is a constant and $\beta=\beta(D)=\left(\left(\sigma_{0}-\sigma_{*}\right)+\sigma_{*} \cdot D\right) /(1-D)$. This is of the Kachanov [1] type.

\section{An experimental illustration}

Usually the empirical criterion for spallation is assumed as [10]

$$
\left(\frac{\sigma_{0}-\sigma_{c_{0}}}{\sigma_{c}}\right)^{k(D)} \cdot t=K(D) .
$$

This expression can be reduced to

$$
\dot{D}=f\left(D, \sigma_{0}\right)
$$

independent of time $t$ explicitly, therefore autonomous.

This may imply a kind of viscous dissipation in damage growth, in accord with the above discussion. Actually, experimental data fitting of the growth rate showed

$$
V \sim\left(c-c_{0}\right)^{\alpha}
$$

$\alpha \simeq 0.8$. Roughly speaking, this does seem to be the viscous dissipation type [7].

\section{Acknowledgements}

This work was supported by the National Natural Science Foundation of China and the Chinese Academy of Sciences under special grant KM85-33.

\section{References}

1. L.M. Kachanov, Introduction to Continuum Damage Mechanics. Martinus Nijhoff, The Netherlands (1986).

2. A.C.F. Cocks and M.F. Ashby, On creep fracture by void growth, Progress in Materials Science 27 (1981) 189-244.

3. Y. Bai, C. Lu, F. Ke and M. Xia, Evolution induced catastrophe, Physics Letters A 185 (1994) 196-200.

4. M.F. Xia, Y.L. Bai and F.J. Ke, Statistical description of pattern evolution in damage-fracture, Science in China A 37, No. 3, 331-340.

5. Y. Bai, F. Ke and M. Xia, Formulation of statistical evolution of microcracks in solids, Acta Mechanica Sinica 7, No. 1 (1991) 59-66.

6. F.J. Ke, Y.L. Bai and M.F. Xia, Evolution of ideal micro-crack system, Science in China A 33, No. 12 (1990) $1447-1459$.

7. W.S. Han, Meso-mechanical laws governing spallation in an Al alloy, Ph.D. Thesis, Institute of Mechanics, Chinese Academy of Sciences, Beijing (1993).

8. L. Davison and A.L. Stevens, Continuum measures of spall damage, Journal of Applied Physics 43, No. 3 (1992) 988-994.

9. D.R. Curran, L. Seaman and D.A. Shockey, Dynamic failure of solids, Physics Reports 147, No. 5-6 (1989) 253-388.

10. L.T. Shen, S.D. Zhao, Y.L. Bai and L.M. Luo, Experimental study on the criteria and mechanism of spallation in an Al alloy, International Journal of Impact Engineering 12 No. 1 (1992) 9-19. 